\title{
Long-term follow-up of Class II adults treated with orthodontic camouflage: A comparison with orthognathic surgery outcomes
}

\author{
Colin A. Mihalik, DDS, MS ${ }^{a}$, William R. Proffit, DDS, PhD ${ }^{b}$, and Ceib Phillips, PhD $^{c}$ \\ aFormer resident, now active duty in U.S. Air Force. Department of Orthodontics, School of \\ Dentistry, University of North Carolina, Chapel Hill. \\ ${ }^{\mathrm{b}}$ Kenan professor. Department of Orthodontics, School of Dentistry, University of North Carolina, \\ Chapel Hill. \\ 'Research professor. Department of Orthodontics, School of Dentistry, University of North \\ Carolina, Chapel Hill.
}

\begin{abstract}
Thirty-one adults who had been treated with orthodontics alone for Class II malocclusions were recalled at least 5 years posttreatment to evaluate cephalometric and occlusal stability and also their satisfaction with treatment outcomes. The data were compared with similar data for longterm outcomes in patients with more severe Class II problems who had surgical correction with mandibular advancement, maxillary impaction, or a combination of those. In the camouflage patients, small mean changes in skeletal landmark positions occurred in the long term, but the changes were generally much smaller than in the surgery patients. The percentages of patients with a long-term increase in overbite were almost identical in the orthodontic and surgery groups, but the surgery patients were nearly twice as likely to have a long-term increase in overjet. The patients' perceptions of outcomes were highly positive in both the orthodontic and the surgical groups. The orthodontics-only (camouflage) patients reported fewer functional or temporomandibular joint problems than did the surgery patients and had similar reports of overall satisfaction with treatment, but patients who had their mandibles advanced were significantly more positive about their dentofacial images.
\end{abstract}

For nongrowing patients with skeletal Class II malocclusions, there are only 2 possible treatment approaches: (1) orthodontic camouflage, based on retraction of the protruding maxillary incisors to improve both dental occlusion and facial esthetics without correcting the underlying skeletal problem; or (2) orthognathic surgery to reposition the mandible or the maxilla. Skeletal Class II problems are due to mandibular deficiency or downwardbackward rotation of the mandible caused by excessive vertical growth of the maxilla. Surgical treatment, therefore, consists of mandibular advancement, superior repositioning of the maxilla, or a combination. Mandibular deficiency is the problem in about two thirds of surgical patients; one third require maxillary surgery, either alone (15\%) or combined with mandibular surgery $(20 \%){ }^{1}$

In one of the few published comparisons of orthodontic versus surgical correction of Class II problems in nongrowing patients, our research group noted that both orthodontic and surgical patients showed similar correction of the malocclusion, although the camouflage group had slightly greater overjet at 1 year posttreatment. ${ }^{2}$ The surgical patients, as

Copyright $(\subseteq 2003$ by the American Association of Orthodontists.

Reprint requests to: William R. Proffit, Department of Orthodontics, School of Dentistry, University of North Carolina, Campus Box 7450, Chapel Hill, NC 27599-7450; William_Proffit@dentistry.unc.edu.. 
expected, had a more ideal skeletal relationship, with the mandible more anteriorly positioned and the mandibular incisors in a more ideal position relative to basal bone.

Better data exist for long-term outcomes for orthognathic surgery than for orthodontic camouflage, although many more Class II patients are treated orthodontically. Surgery patients have been followed carefully because of concerns about condylar remodeling or other skeletal changes that would produce relapse, but dental and skeletal stability in nonsurgical patients has not been evaluated in the same systematic way. Existing data show that during the first postsurgical year, both mandibular advancement and maxillary intrusion are quite stable in most surgical Class II patients. At 1 year, skeletal landmarks for both groups were within $2 \mathrm{~mm}$ of the immediate postsurgical position in more than $90 \%$ of the patients in the University of North Carolina (UNC) database, and similar results have been reported from other centers. Comparable stability for 2-jaw surgery requires rigid internal fixation, but, with it, more than $90 \%$ of the patients are stable during the first year. ${ }^{3}$

Skeletal changes occur in a surprising number of surgery patients between 1 and 5 years postsurgery, however. Long-term (5-year) recall of UNC patients who had mandibular advancement showed a small but statistically significant decrease in mean mandibular length (condylion-Point B) from 1 to 5 years. Usually after any type of treatment, a few patients have most of the changes, so that a better perspective is gained from noting that, at 5 years postsurgery, the mandible is within $2 \mathrm{~mm}$ of its immediate postsurgical position in $80 \%$ of the patients, and $5 \%$ have more than $4 \mathrm{~mm}$ shortening of mandiblar length $(\mathrm{Co}-\mathrm{Pg}){ }^{4,5}$ These skeletal changes are not necessarily accompanied by occlusal relapse, because compensatory movement of the teeth often occurs.

To compare this with long-term skeletal changes in adults who did not have surgery, Schubert et $\mathrm{al}^{6}$ recalled 33 adults for whom cephalometric radiographs were available at the end of orthodontic treatment. Of these patients, 9 had refused recommendations for surgery ( 2 chose orthodontics), and 24 received orthodontic treatment that would have produced dental but not skeletal changes; ie, they had milder malocclusions and did not require surgery. Although evidence of skeletal remodeling over a 5-year period was observed in these orthodontic patients, surgery patients had larger changes in most landmark positions and were more likely to have a decrease in mandibular length. Because both the magnitude and the pattern of change in the nonsurgical adults were different from the surgery patients, the changes beyond 1 year postsurgery could not be attributed just to normal adult growth. There are no data for long-term changes in a sample of only adult Class II patients treated with orthodontics alone.

Both occlusal and skeletal stability are important in comparing treatment outcomes. The peer assessment rating (PAR) method developed in England provides a weighted measure of the occlusion that takes into account overjet, overbite, midlines, buccal occlusion, and maxillary and mandibular anterior alignment. ${ }^{7} \mathrm{Al}$ Yami et al ${ }^{8}$ used PAR scores to evaluate the treatment outcomes 10 years after treatment in 564 adolescents (mean age, 12.0 years \pm 3.1 years at the start of treatment) with various orthodontic problems. Their results showed that about two thirds of the achieved orthodontic correction was still present 10 years after treatment. When PAR scores increased after treatment, about half of the change occurred within the first 2 years. Otuyemi and Jones ${ }^{9}$ used PAR scores to characterize the relative success of orthodontic treatment in adolescent Class II patients and found on recall that $60 \%$ remained in the same category at 1 year and $38 \%$ at 10 years; the major factors in higher scores were an increase in overjet and a deterioration of mandibular anterior alignment.

In evaluating treatment outcomes, not only the objective changes measured from radiographs and other records but also the patients' perceptions must be noted. A major 
reason for seeking orthodontic or surgical correction of a Class II problem is to improve the quality of life, and this must be evaluated from the patient's point of view, not from what the doctor measures on physical records. Most published data on perceptions of treatment are for surgical patients. Although camouflage patients do not undergo orthognathic surgery, they still have teeth extracted, their dental and facial appearance changes, their jaw functions are affected, and the same methods used to study surgery patients can be applied.

Essentially all long-term studies show that most surgical patients are satisfied with their results and would recommend surgery to others. The major sources of information are a series of studies carried out in Seattle in the 1980 s by Kiyak et $\mathrm{a}^{10}$ and studies at UNC in the 1990s by Phillips and Proffit. ${ }^{11}$ In both centers, $75 \%$ to $80 \%$ of patients reported satisfaction with their treatment at 4 to 6 weeks postsurgery, and, by 2 years, nearly $90 \%$ were satisfied. Overall, patients were quite pleased with the esthetic results of treatment, and, even at 2 years postsurgery, many reported receiving positive comments about their appearance. In the UNC group, one fourth of the patients at 2 years postsurgery agreed that people whom they met for the first time reacted more positively than new acquaintances had before the surgery. However, $15 \%$ said they had expected more change in their appearance than achieved, even though they reported satisfaction with the results. No data exist to compare long-term perceptions and feelings of camouflage patients with surgical patients.

This project was based on long-term recall of adult Class II patients who had undergone orthodontic camouflage. It had 3 goals: (1) to compare long-term skeletal and soft tissue changes in these orthodontic patients with surgery patients; (2) to evaluate long-term changes in occlusal relationships after orthodontic camouflage and compare occlusal stability with surgery patients; and (3) to evaluate patient satisfaction after camouflage treatment by using a similar survey instrument to that previously used with surgery patients.

\section{MATERIAL AND METHODS}

Despite an underlying skeletal problem, orthodontic camouflage treatment, by definition, is based on tooth movement to correct only the dental occlusion. It typically requires premolar (occasionally other) extractions. For this study, we reviewed charts for all 135 patients in the UNC orthodontic clinic database who had orthodontic treatment between 1980 and 1995 for Class II malocclusion beginning when minimal growth would be expected (women, age 17 or over; men, age 19 or older) that involved dental extractions. Of this group, 100 completed orthodontic treatment at UNC ( 27 moved, transferred, or failed to complete treatment, and 8 had orthognathic surgery). Two of the 100 had died, and 24 did not have complete records including 1-year recall. The remaining 74 patients were selected for potential recall.

An extensive search (with Internet-based locating resources) was made to locate these patients, and 33 were found. The patients who could not be contacted had a mean age of 26.8 years (SD 8.00), 82\% were female, and they had $5.7 \mathrm{~mm}$ mean overjet at the start of treatment (SD 2.68). The 31 who were recalled were very similar before treatment (Tables I and II). Once the patients were contacted, they were willing to return to participate in the study, especially if their travel costs were reimbursed. Only 2 patients declined, and both cited distance as a hindrance. Of the 31 recalled ( $42 \%$ of those originally selected), the average length of time since the end of orthodontic treatment was 12 years (range, 6.5 to 15.7 years). From the original sample of 74 patients, $85 \%$ were female, but 30 of the 31 recalled patients $(97 \%)$ were female.

On recall, complete clinical records (impressions for study casts, intraoral and extraoral photographs, panoramic and lateral cephalometric radiographs, recall data forms) were 
obtained, and the patients completed questionnaires on perception of current problems and satisfaction (described below).

The long-term cephalometric changes of interest were those between 1 year posttreatment and the long-term recall. For both cephalometric and perception data, these adult orthodontic camouflage patients were compared with 3 groups of Class II orthognathic surgical patients for whom long-term data had been previously published. ${ }^{3-5}$ Pretreatment characteristics of the 4 groups are summarized in Tables I and II. In addition, cephalometric changes were compared with the adult sample of Shubert et $\mathrm{al}^{6}$ with long-term follow-up after no treatment or orthodontic treatment for mild problems. All camouflage subjects were treated in the Department of Orthodontics at UNC, and all surgery patients had their surgeries at UNC. Approximately $25 \%$ of this group also had the orthodontic phase of their treatment at UNC; the others were treated in private practices.

As in previous studies at UNC, cephalometric radiographs for the Class II camouflage patients were digitized by using the UNC 139 -point model. ${ }^{2}$ A coordinate system was established, with a line through sella rotated $6^{\circ}$ down anteriorly from the $\mathrm{SN}$ line as the horizontal axis, and a vertical line through sella perpendicular to it as the vertical axis. Angular measures and millimeter changes in landmark position were obtained as coordinate changes in this reference system. Thus, changes relative to the $x, y$-axis could be compared with previous studies at UNC of surgery and adult orthodontic patients. Cephalograms for all subjects in all treatment groups were obtained at UNC and digitized by the same research staff personnel.

In addition to descriptive statistics, analysis of covariance (ANCOVA) was used to estimate the average differences between the camouflage group and the surgically treated groups. Baseline values, age at the start of treatment, and the duration of follow-up were included as covariates, and adjusted mean change was compared among the 3 surgical groups and the Class II camouflage groups. ANCOVA was used to correct for the potential bias because of the differences in mean baseline value, age, and length of follow-up between the groups, and because of the possible relationships between the means of these covariates and the mean cephalometric changes in the treatment groups. ANCOVA removes this bias as far as possible statistically by comparing the mean change in the treatment groups at the same relative mean baseline value, age, and length of follow-up.

PAR scores were calculated from the dental casts for the camouflage patients at the end of treatment and on long-term recall, using both the English and the American weightings.

At the recall appointment, 27 of the 31 orthodontic Class II patients completed 3 questionnaires developed to evaluate perceptions in orthognathic surgery patients. ${ }^{11}$ The facial-image (FI) scale is a 16-item questionnaire that asks the respondent to rate each facial feature from (1) have strong negative feelings to (5) have strong positive feelings. Two subscales are scored. The dentofacial subscale is sensitive to appearance changes that can be affected by different treatment approaches, and the cosmetic subscale includes facial features that are not affected by orthodontic or orthognathic treatment.

The second questionnaire, problems with occlusion and function (POF), consists of 21 statements and has a 1-to-5 agree or disagree format. Two subscales are scored: functional concerns and temporomandibular joint (TMJ) concerns. The third questionnaire, satisfaction (SAT), is a 16-item instrument on which 3 subscales are scored: (inter)personal outcome; functional outcome; and preparation/knowledge satisfaction. The item content for the preparation/knowledge subscale was modified slightly from that used for the surgery patients, because preparation for surgery did not apply to the orthodontic camouflage 
patients. Each item was presented as a statement rated on a 7-point scale from "disagree strongly" to "agree strongly."

The camouflage patients were compared with all UNC Class II surgical patients who had completed long-term perception survey instruments (who were not exactly the same groups for the cephalometric comparison). ${ }^{6}$ The long-term mean subscale responses of the surgery and the camouflage groups were compared with the Cochran-Mantel-Haenszel row mean score test.

\section{RESULTS}

Mean cephalometric changes for the camouflage patients during treatment and on long-term recall are shown in Figures 1 and 2, and tabulated data for changes from 1-year posttreatment to long-term recall are shown in Table III. As one would expect, the treatment changes were mainly retraction of the maxillary incisors. Long-term changes were quite small. Eruption of the mandibular incisors, leading to an average increase in overbite of 1.5 $\mathrm{mm}$, was the largest mean change.

In clinical studies, a few patients usually show most of the change, so descriptive statistics based on the normal distribution can be misleading. For the camouflage patients, however, significant changes in skeletal landmarks were not observed. No patient had more than 2 $\mathrm{mm}$ change in Points A or B or pogonion. The cephalometric data for the camouflage patients showed almost no long-term relapse changes except for over-bite. Pearson correlation coefficients showed no relationship between the length of time to recall and the changes from end of treatment to follow-up.

PAR scores for the camouflage patients at the end of treatment and on long-term recall are shown in Tables IV, and PAR weightings in the 2 systems are given in Table V. The modest long-term increase indicates generally good stability of the occlusal result.

As Table III shows, the differences in mean long-term changes among the camouflage, general treatment, and surgical groups were small. Analysis of covariance showed, however, that there was a statistically significant difference in the change in mandibular landmark positions between the orthodontic and surgical groups. In all groups, if it changed at all, the mandible tended to come forward and downward, but there was a significantly greater chance of movement (growth?) in the nonsurgical groups.

This is clarified by Figures 3 through 5, showing the percentages of patients in each group with more than $2 \mathrm{~mm}$ change in landmark positions or dimensions. Note (Fig 3) that backward movement of the chin and Points A and B occurred only in the surgery groups, but forward movement of Point B and Pg also occurred in all 3 surgical groups, and forward movement of $\mathrm{Pg}$ was as likely as backward movement after mandibular advancement. Forward movement of all 3 landmarks and of the incisors occurred in 15\% of the general treatment patients. In the camouflage patients, who were remarkably stable, the only change was backward movement of the mandibular incisors in 10\%. What is not in Figure 3 is important: only for the mandibular incisor position did patients in the camouflage group have any long-term changes greater than $2 \mathrm{~mm}$, and so they do not show up in the other sections of this figure, although the data were carefully examined for changes of this magnitude.

Long-term vertical changes occurred in more surgery than orthodontic patients (Fig 4), with one fourth of the patients who had maxillary surgery showing downward movement suggesting continued vertical growth. Incisor changes were smaller than skeletal changes. Overjet was stable in both orthodontic groups (Fig 5) and showed a long-term increase in 
$10 \%$ of the 2-jaw surgery patients, in $15 \%$ of the maxillary impaction group, and in $20 \%$ of the mandibular advancement patients. In contrast, overbite increased in $10 \%$ to $15 \%$ of the patients in both the orthodontic and the surgery groups. The percentage with overjet changes was much greater than the percentage with changes in the ANB angle; ie, this long-term change in the surgery groups was due more to tooth movement than skeletal change. The greater change in mandibular plane angles for the surgery patients is consistent with continued remodeling at gonion after surgery. Mandibular length, as measured from condylion to pogonion, decreased in 5\% of the orthodontic patients and increased in $10 \%$. The percentage of surgery patients with a decrease in length was greater, but nearly as many surgery patients had an increase in mandibular length as a decrease.

Results from the perception survey instruments are given in Tables VI through XI, which show the percentages of patients with a strongly positive response (5-7 on the 7-point scale of SAT, and 4-5 on the 5-point scales of POF and FI). Although the average subscale score is the best summary of patients' perceptions of a specific domain or construct, the percentage of positive responses for each item can illustrate differences in the pattern of responses that might result in the same average score. Surgery patients were asked about their perceptions of the surgical experience, but orthodontic patients obviously were not; thus all items on the 2 SAT versions could not be compared.

SAT scores for the camouflage patients were quite high (Table VI and VII). Perhaps the most sensitive indicator of satisfaction is reflected by willingness to undergo the same treatment again after knowing how it turned out; $92 \%$ of the camouflage group were strongly positive. Nearly all were pleased with the change in their appearance, but only one third agreed strongly that they could eat more easily.

Comparison with the surgery groups shows greater long-term satisfaction for the camouflage patients in almost every category, despite the positive numbers for satisfaction in the surgery groups. The only statistically significant difference, however, was that the maxillary impaction surgery group was less positive than the other groups for the interpersonal outcomes subscale (Cochran-Mantel-Haenszel statistics based on table scores, $P<.001$ ).

Only a few of the camouflage patients had functional or pain problems (Tables VIII and IX). Although 15\% thought that their front teeth did not fit properly, no patients complained that they could not chew food well. Of the $18 \%$ of the camouflage patients who reported pain in their TMJs, over half reported pain in jaw muscles as well.

These numbers also compare favorably with those of the surgery patients. Scores for occlusion and function were similar, except that the maxillary surgery patients were more likely to feel that they could not chew food well. In all 3 surgery groups, reports of TMJrelated problems and pain or discomfort were 2 or 3 times more prevalent than in the camouflage group, and the difference between the surgery and camouflage patients was statistically significant $(P<.01)$.

The facial image can be broken into 2 subcategories - cosmetic and dentofacial images (Tables X and XI). Half to two thirds of the camouflage patients responded quite positively to the cosmetic image items (that their orthodontic treatment would not have influenced), and the frequency of positive responses was only slightly greater for the dentofacial items that would have been affected. For the mouth, half $(48 \%)$ of the camouflage group gave the highest score of 5 on the 5-point scale, and $41 \%$ scored their teeth at 5.

On the cosmetic scale, the camouflage patients' frequency of positive items ( 4 and 5 on a $1-5$ scale) was scored at or slightly below the level for the surgery patients. Facial skin tone was rated most positively by the maxillary impaction group and least positively by the 
camouflage group, although hard tissue support for the soft tissue facial mask would have been decreased by both types of treatment. Intermediate scores were reported by the patients who had mandibular advancement, which would increase soft tissue support and should have tightened the skin. Advancing a deficient mandible improves chin and neck contours, and the mandibular advancement patients reported higher positive feelings in this area.

For the dentofacial scale, both groups of patients who had mandibular advancement were much more positive about their chin and profile than were the camouflage patients, as one would think they should have been, and this difference was statistically significant $(P<$. 005). There was no difference between the maxillary impaction and the camouflage patients.

\section{DISCUSSION}

Patients treated with orthodontic camouflage have, in general, less severe problems than those treated surgically. These data do not report outcomes of alternative treatment for comparable problems. As Table II shows, the camouflage patients that we studied had less severe malocclusions initially (overjet and overbite) and smaller jaw discrepancies than the surgery patients. Despite the initial differences, jaw relationships and dental occlusion were similar at the end of treatment, so both types of treatment largely met their treatment objectives. The amount of change produced by treatment was larger in the surgical groups, and they experienced a component of skeletal change that the orthodontic patients did not. The greater amount of treatment change in the surgery groups probably contributed to the greater prevalence of posttreatment change.

When only part of a group of treated patients can be recalled, those who cooperated with the recall might not truly represent all patients who underwent treatment. In this study, it was possible to recall $42 \%$ of the Class II camouflage patients with complete records that included the 1-year recall; they represented $31 \%$ of those who had appropriate treatment completed at UNC. Because all but 2 of the patients who were located agreed to return, willingness to participate probably did not bias the reported perceptions or physical outcomes. The recall percentages for long-term postsurgery patients are similar to those for the camouflage group. Although there is no way to be certain that patients who can be located are precisely comparable with those who cannot, we have found no reason to think that their perceptions or outcomes are systematically different.

Cephalometrically, the camouflage patients were quite stable in the long term, although $12 \%$ had an increase in overbite that could be attributed to the eruption of the mandibular incisors. Relapse of more than a 2-mm long-term increase in overjet was not observed. The amount of long-term change was not significantly related to the length of time to recall, which in any case was longer for the camouflage patients than for the groups to which they were compared.

The percentage of patients with an increase in overbite was almost identical in the surgery and camouflage groups, but an increase in overjet occurred in $10 \%$ to $20 \%$ of the surgery patients. Because for them skeletal change rather than tooth movement was the major factor in reducing overjet through treatment, it is surprising that the posttreatment changes were as likely to be due to tooth movement as skeletal relapse. Perhaps this reflects greater changes in relationships between the incisors and the facial soft tissue mask in the surgery group, but the surgery patients were stable during the first posttreatment year and showed changes after that, and soft tissue pressure changes would be expected to affect tooth position more quickly during the first year. Previous reports have noted that skeletal changes in surgery patients are not necessarily reflected in the occlusion, ${ }^{3-5}$ and changing dental and soft tissue 
relationships probably contributes to dental compensation for skeletal change when it occurs.

Not all the skeletal changes in these adults were in a relapse direction. Continued growth of the facial skeleton has been appreciated for years now, but it still is surprising to see how much long-term change can occur in ways consistent with continued growth in patients who are expected to have little or no growth potential. Forward movement of facial landmarks was observed in $15 \%$ of the long-term camouflage group, and one fourth to one third of the surgery patients showed what appeared to be growth changes, ie, downward movement of the maxilla or forward movement of the mandible. Do the data suggest that late growth is more likely in patients with the severe problems that are selected for surgery, or is this another reflection of skeletal responses to greater soft tissue changes in surgery patients? We cannot be sure at present.

For occlusal stability, mandibular incisor alignment is the weak link, and retainer wear is obviously an important factor. This study showed that slightly over half of the camouflage patients had a long-term increase in incisor irregularity over time. Comparable data for the surgery patients were not available. $\mathrm{Al} \mathrm{Yami} \mathrm{et} \mathrm{al}{ }^{8}$ showed that the $11 \%$ of their large sample who had bonded lower retainers had better alignment at 5 and 10 years posttreatment even if their initial PAR scores were higher. In our camouflage sample, 10 of 31 patients (32\%) with bonded lower retainers were present on long-term recall. Of these, 3 had minor slippages of 1 or 2 contacts, and the rest had no change in this component of the PAR score.

The extent to which removable retainer wear influenced changes in PAR scores is difficult to determine, but it seems likely that this would have an effect. Two camouflage patients specifically stated that they wore their removable retainers only for a few months and then stopped because of "the hassle," but 3 patients were still wearing both upper and lower removable retainers every night, 10 years or more out of treatment.

In evaluating patients' perceptions of treatment outcomes, it also is potentially important that the camouflage group was so overwhelmingly female. Most older Class II patients in the orthodontic clinic are women (relatively more women receive late Class II treatment than adolescent or preadolescent treatment), and all but 1 of those who were recalled were female. Most Class II surgery patients are also female, but the surgery groups were $25 \%$ to $30 \%$ male. In the surgery groups, no sex difference in response to the survey instruments has been detected. We do not know whether this also would be true for camouflage patients.

The camouflage group overall was very satisfied with their treatment and had fewer functional and TMJ problems than any surgical group. They were positive about aspects of facial appearance that were not affected by treatment as well as those that were; in most respects, they were as positive as the surgery patients, except that the mandibular advancement group had $20 \%$ to $25 \%$ more patients feeling positive about their chins. Similar findings were reported by Bell et $\mathrm{al}^{12}$ in the $1980 \mathrm{~s}$, who noted that even though Class II camouflage patients were aware of differences in chin projection, most were pleased with the outcome of treatment.

From our perspective, these data show that properly selected patients for orthodontic camouflage treatment are as likely or more likely to be satisfied with the outcome of treatment as those who have surgery. Proper selection of patients, of course, is neither simple nor easy. When alternative treatment approaches are possible, the ratio of benefit to risk must be considered for each procedure. For orthodontic camouflage versus surgery, the important decision is whether the greater improvement in dentofacial image that is possible with surgery would be worth the increased cost and risk. The risks of surgery obviously can be much greater than those of a nonsurgical approach. The most common surgical risk is 
decreased sensation of the lips, whereas the greatest risk for camouflage patients appears to be resorption of the maxillary incisor roots as they are retracted and torqued against the lingual cortical plate. The ideal patient for camouflage should have reasonably good facial esthetics initially, with overjet created more by maxillary incisor protrusion than mandibular retrusion. The more severe the mandibular deficiency and the greater the overjet, the greater the need for surgery to obtain satisfactory clinical correction.

How patients perceive the severity of their problems is a factor in the decision to have surgery rather than camouflage or no treatment. There is good evidence that the more the person perceives herself (or himself) as normal, the more likely he or she is to choose orthodontics alone and to be satisfied with the outcome. Conversely, patients who perceive themselves as outside the normal range are more likely to prefer surgery and to be dissatisfied with tooth movement alone. ${ }^{11}$ Problem severity cannot be evaluated just from cephalometric radiographs, dental casts, and other physical records. Because of these perception effects, we cannot set physical characteristics as the sole definition of whether surgical correction is required or whether orthodontics alone would be satisfactory.

When alternative treatments are available, the doctor should provide the information a patient needs to make an informed decision. Use of computer image predictions of outcomes can help patients visualize the effect of surgery versus camouflage, and existing data now show that the benefits of this approach outweigh the risks of arousing patients' unrealistic expectations. ${ }^{12,13}$ This is an excellent way to involve the patient in the decision as to the preferred mode of treatment and to evaluate what the patient perceives - an essential part of proper selection of patients for camouflage or surgery.

\section{Acknowledgments}

We thank Ms Debora Price for help with data acquisition and analysis, and Drs George Blakey and L'Tanya Bailey for helpful comments.

Supported in part by NIH grant DE-05215 from the National Institute of Dental and Craniofacial Research.

\section{REFERENCES}

1. Proffit, WR.; White, RP.; Sarver, DM. Contemporary treatment of dentofacial deformity. Mosby; St. Louis: 2003.

2. Proffit WR, Phillips C, Douvartzidis N. A comparison of outcomes of orthodontic and surgicalorthodontic treatment of Class II malocclusion in adults. Am J Orthod Dentofacial Orthop. 1992; 101:556-65. [PubMed: 1598896]

3. Proffit WR, Turvey TA, Philips C. Orthognathic surgery: a hierarchy of stability. Int J Adult Orthod Orthognath Surg. 1996; 11:191-204.

4. Simmons KE, Turvey TA, Phillips C, Proffit WR. Surgical-orthodontic correction of mandibular deficiency: five-year follow-up. Int J Adult Orthod Orthognath Surg. 1992; 7:67-80.

5. Miguel JA, Turvey TA, Phillips C, Proffit WR. Long-term stability of two-jaw surgery for treatment of mandibular deficiency and vertical maxillary excess. Int J Adult Orthod Orthognath Surg. 1995; 10:235-45.

6. Schubert P, Bailey LJ, White RP, Proffit WR. Long-term cephalometric changes in untreated adults compared to those treated with orthognathic surgery. Int J Adult Orthod Orthognath Surg. 1999; 14:91-9.

7. Richmond S, Shaw WC, Roberts CT, Andrews M. The PAR index (Peer Assessment Rating): methods to determine outcome of orthodontic treatment in terms of improvement and standards. Eur J Orthod. 1992; 14:180-7. [PubMed: 1628684] 
8. A1 Yami EA, Kuijpers-Jagtman AM, Van't Hof MA. Stability of orthodontic treatment outcome: follow-up until 10 years postretention. Am J Orthod Dentofacial Orthop. 1999; 115:300-4. [PubMed: 10066979]

9. Otuyemi OD, Jones SP. Long-term evaluation of treated Class II division 1 malocclusions utilizing the PAR index. Brit J Orthod. 1995; 22:171-8. [PubMed: 7640256]

10. Kiyak, HA.; Bell, R. Psychosocial considerations in surgery and orthodontics.. In: Proffit, WR.; White, RP., Jr, editors. Surgical-orthodontic treatment. Mosby; Saint Louis: 1991.

11. Phillips, C.; Proffit, WR. The psychosocial effect of dentofacial deformity.. In: Proffit, WR.; White, RP.; Sarver, DM., editors. Contemporary treatment of dentofacial deformity. Mosby; St. Louis: 2002.

12. Bell R, Kiyak HA, Joondeph DR, McNeill RW, Wallen TR. Perceptions of facial profile and their influence on the decision to undergo orthognathic surgery. Am J Orthod. 1985; 88:323-32. [PubMed: 3863490]

13. Phillips C, Hill BJ, Cannac C. The influence of video imaging on patients' perceptions and expectations. Angle Orthod. 1995; 65:263-70. [PubMed: 7486240]

14. Kiyak HA, Zeitler DL. Self-assessment of profile and body image among orthognathic surgery patients before and two years after surgery. J Oral Maxillofac Surg. 1988; 46:365-71. [PubMed: 3163369] 


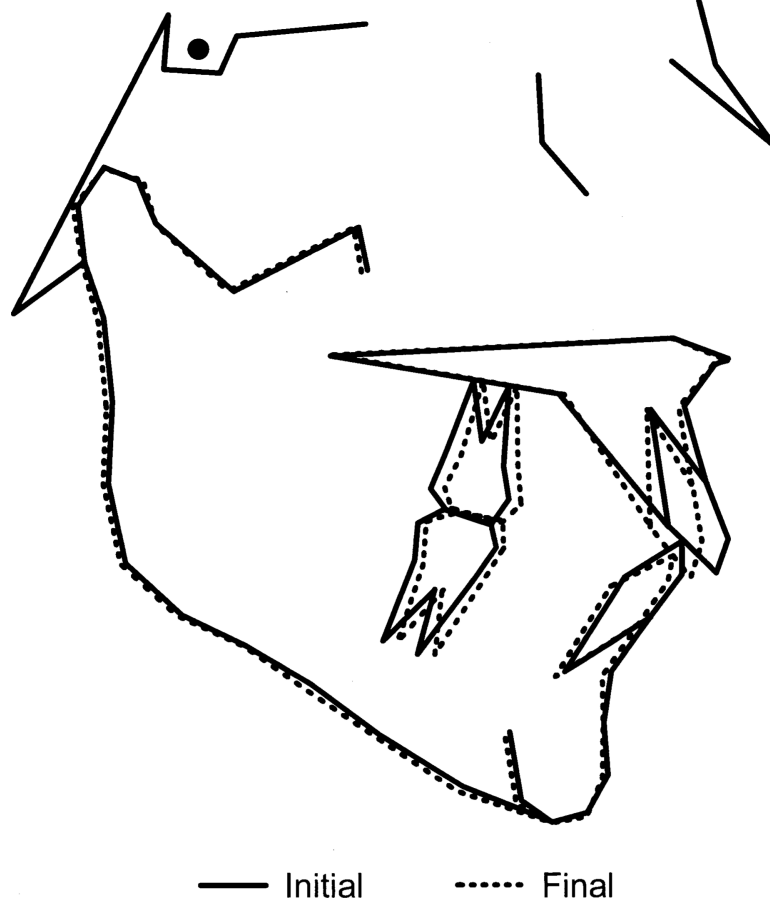

Fig 1.

Mean cephalometric changes, camouflage group, during treatment. 


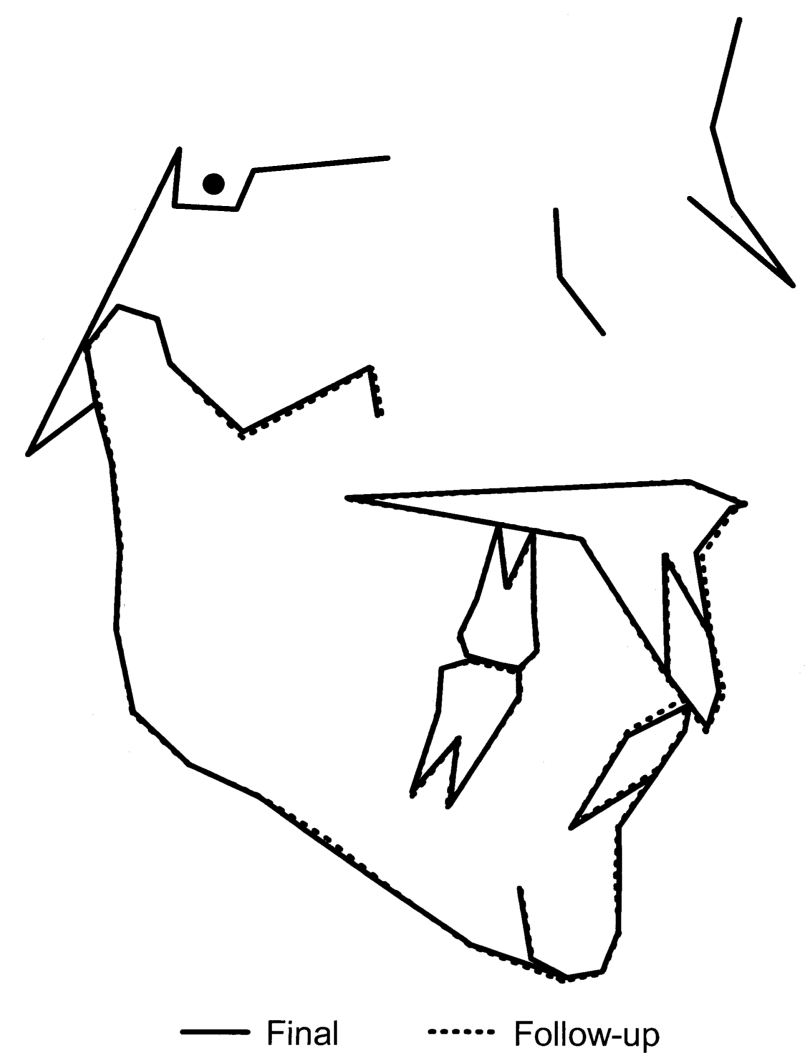

Fig 2.

Mean cephalometric changes, camouflage group, end of treatment to long-term recall. 
$\mathrm{Pg}$

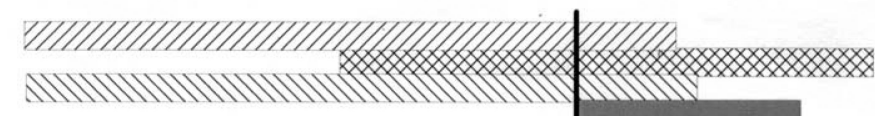

$\mathrm{APt}$

B Pt
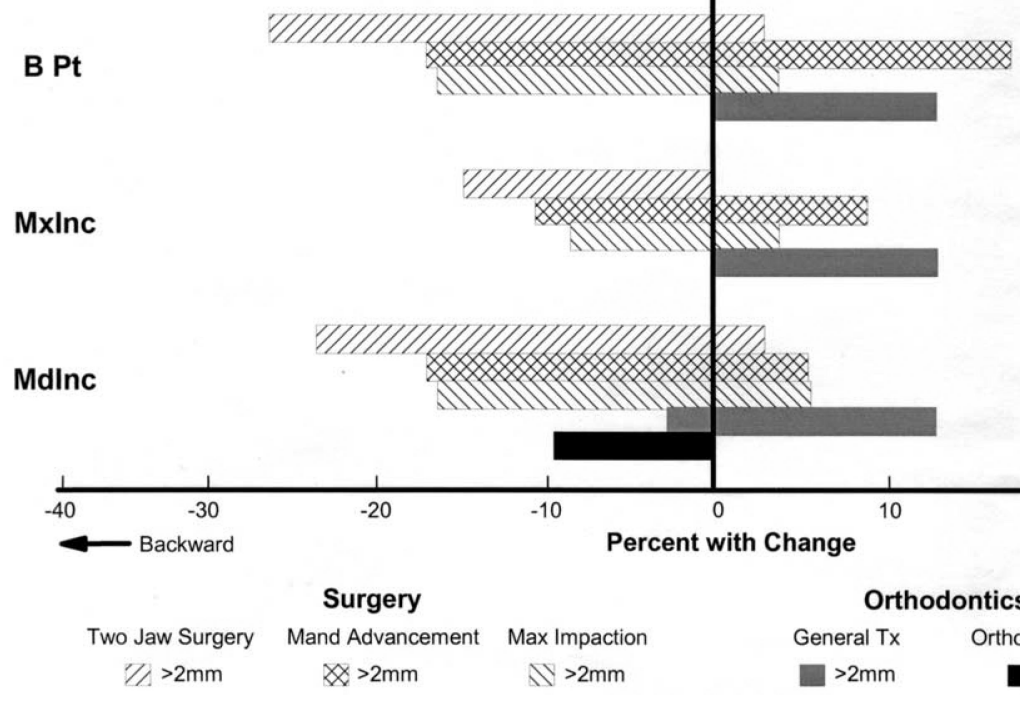

Fig 3.

Percentage of patients in each group with $>2 \mathrm{~mm}$ change in horizontal landmark positions from end of treatment to long-term recall. No camouflage patient had $>2 \mathrm{~mm}$ change. 


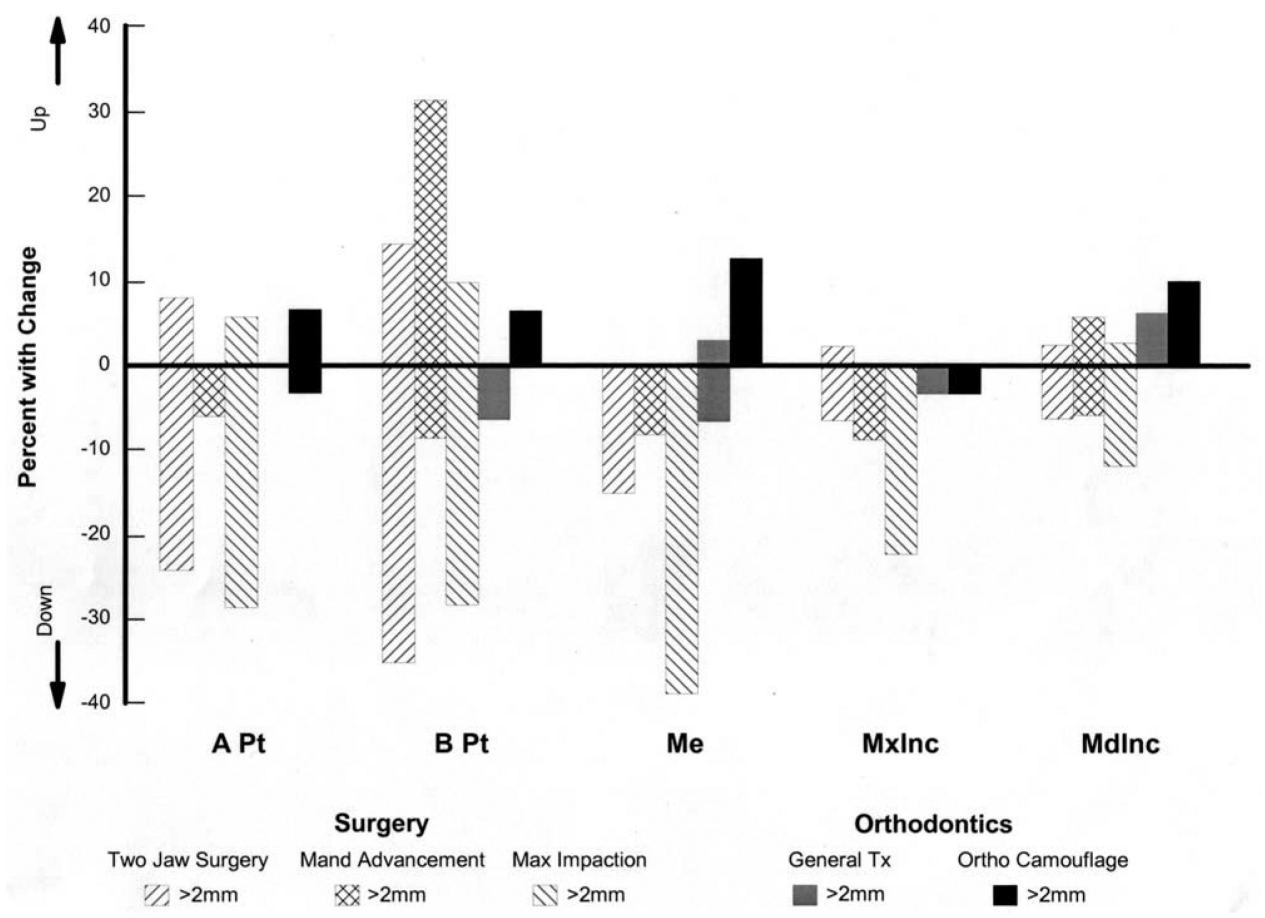

Fig 4.

Percentage of patients with $>2 \mathrm{~mm}$ change in vertical landmark positions from end of treatment to long-term recall. 


\section{Overjet}

Overbite

MdPI

ANB

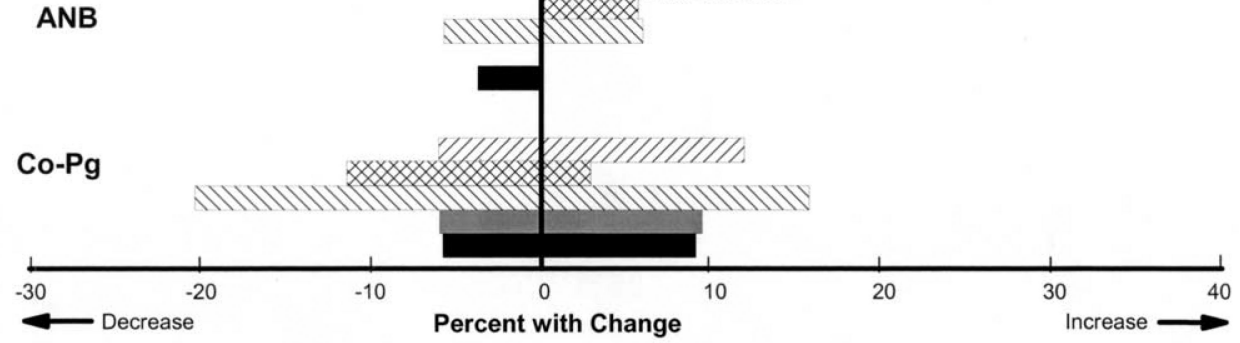

Surgery

Two Jaw Surgery Mand Advancement Max Impaction $1 / />2 \mathrm{~mm}$
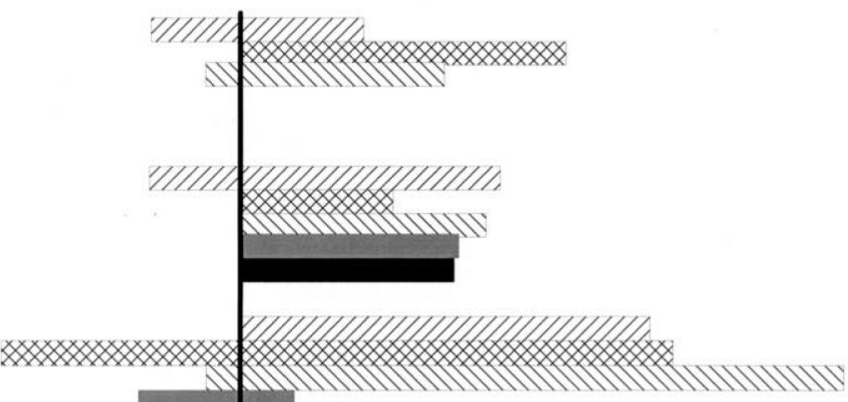

Fig 5.

Percentage of patients with $>2 \mathrm{~mm}$ change in dimensions from end of treatment to long-term recall. 


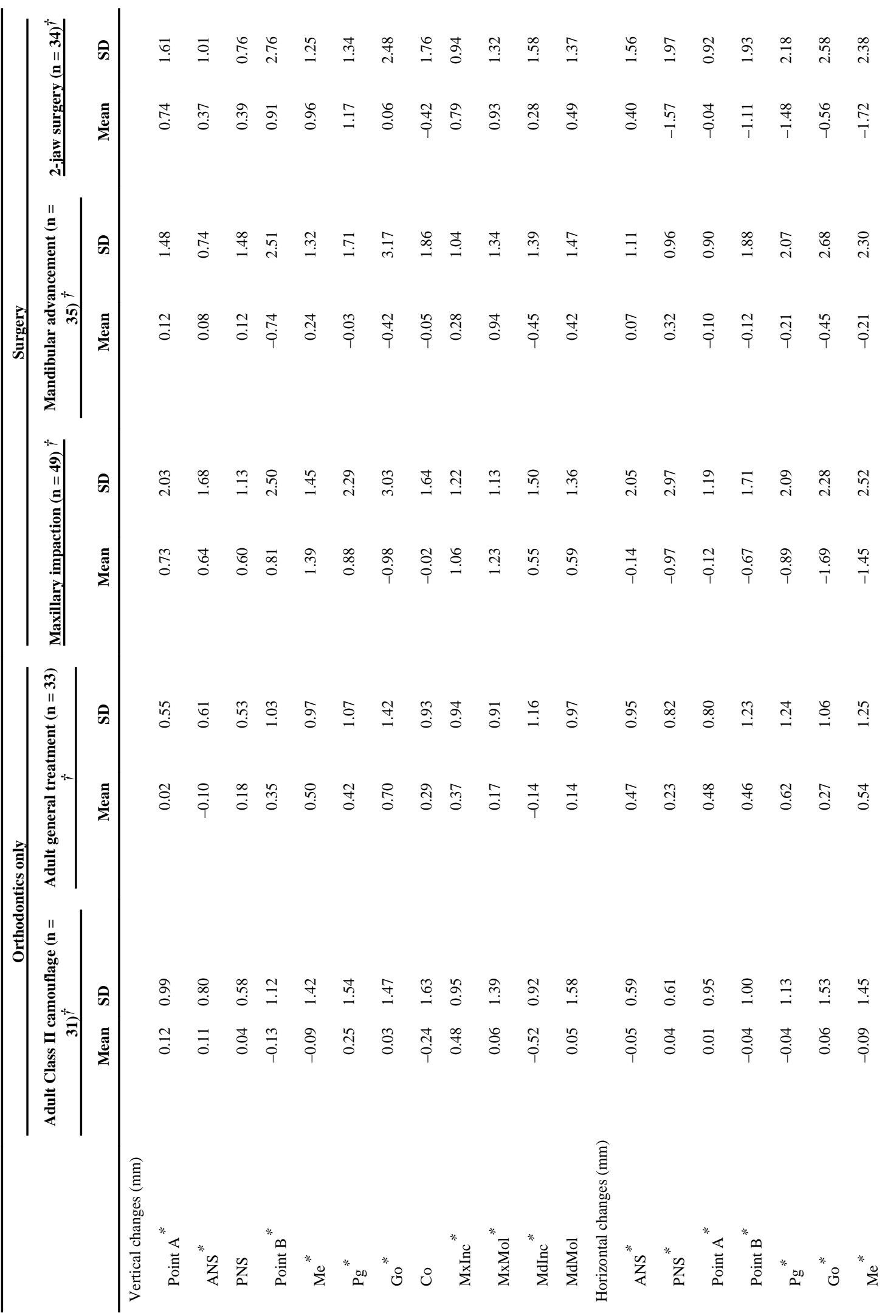




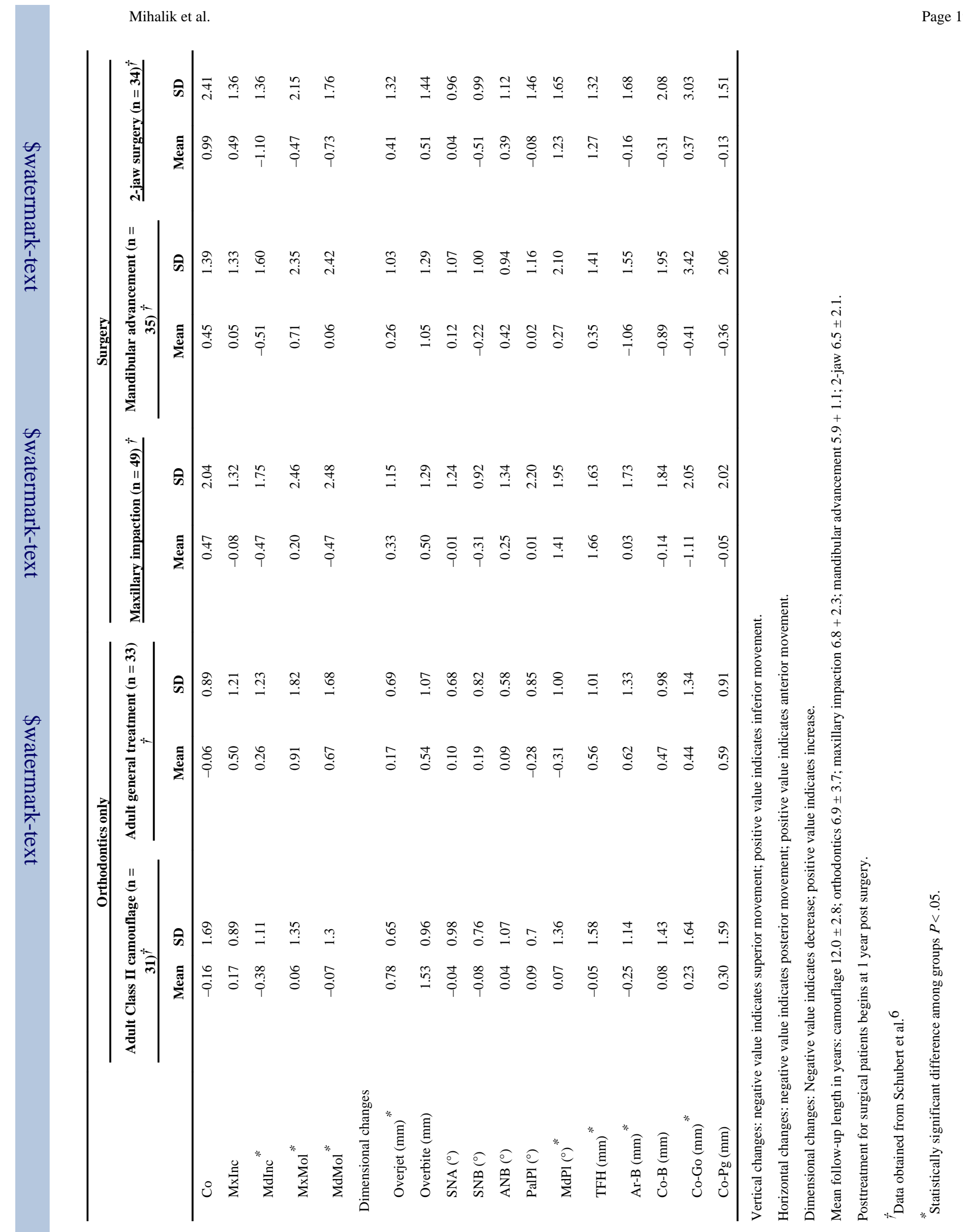

Am J Orthod Dentofacial Orthop. Author manuscript; available in PMC 2013 January 26. 
Table IV

Mean PAR scores in Class II camouflage group

\begin{tabular}{lccrc}
\hline & End treatment & SD & Long term & SD \\
\hline Weighted total: & & & & \\
English & 6.14 & 4.39 & 9.55 & 6.10 \\
American & 8.55 & 5.21 & 10.97 & 6.64 \\
English weighted components: & & & \\
UA & 0.27 & 0.53 & 0.80 & 1.00 \\
LA & 0.48 & 0.69 & 1.30 & 1.60 \\
RB & 1.48 & 0.95 & 1.45 & 0.95 \\
LB & 1.62 & 0.82 & 1.48 & 1.06 \\
OJ & 1.86 & 2.82 & 3.31 & 4.12 \\
OB & 0.30 & 0.90 & 0.83 & 1.14 \\
ML & 0.10 & 0.70 & 0.40 & 1.20 \\
\hline
\end{tabular}

$U A$, Upper arch; $L A$, lower arch; $R B$, right buccal; $L B$, left buccal; $O J$, overjet; $O B$, overbite; $M L$, midline. 
Table V

PAR weighting

\begin{tabular}{ccc}
\hline & English & American \\
\hline UA & $\times 1$ & $\times 1$ \\
LA & $\times 1$ & not scored \\
RB & $\times 1$ & $\times 2$ \\
LB & $\times 1$ & $\times 2$ \\
OJ & $\times 6$ & $\times 5$ \\
OB & $\times 2$ & $\times 3$ \\
ML & $\times 4$ & $\times 3$ \\
\hline
\end{tabular}

$U A$, Upper arch; $L A$, lower arch; $R B$, right buccal; $L B$, left buccal; $O J$, overjet; $O B$, overbite; $M L$, midline. 
Table VI

Selected questionnaire items from SAT index, percent at extreme (strongly positive)

\begin{tabular}{|c|c|c|c|c|}
\hline & \multirow{2}{*}{$\begin{array}{r}\text { Orthodontics only } \\
\text { Adult Class II } \\
\text { camouflage }(n=27)\end{array}$} & \multicolumn{3}{|c|}{ Surgery } \\
\hline & & $\begin{array}{c}\text { Maxillary } \\
\text { impaction }(n= \\
\text { 24) }\end{array}$ & $\begin{array}{c}\text { Mandibular } \\
\text { advancement }(n= \\
103)\end{array}$ & $\begin{array}{c}\text { 2-jaw surgery } \\
\quad(n=66)\end{array}$ \\
\hline \multicolumn{5}{|l|}{ Interpersonal outcomes } \\
\hline Receive positive comments since treatment & 69 & 46 & 52 & 67 \\
\hline Pleased with change in appearance & 85 & 63 & 77 & 83 \\
\hline Treatment change was exactly what I expected & 88 & 46 & 63 & 46 \\
\hline People react more positively at initial meeting & 50 & 29 & 30 & 33 \\
\hline Delighted how much better I look & 88 & 42 & 69 & 69 \\
\hline Like what I see in the mirror & 88 & 50 & 76 & 75 \\
\hline \multicolumn{5}{|l|}{ Functional outcomes } \\
\hline Eating is much easier & 35 & 46 & 57 & 53 \\
\hline Can Chew Better & 35 & 54 & 62 & 50 \\
\hline \multicolumn{5}{|l|}{ Other } \\
\hline Recommend treatment to others & 100 & 75 & 87 & 83 \\
\hline Undergo treatment again & 92 & 79 & 85 & 76 \\
\hline Treatment was a positive experience & 100 & 84 & 87 & 88 \\
\hline
\end{tabular}




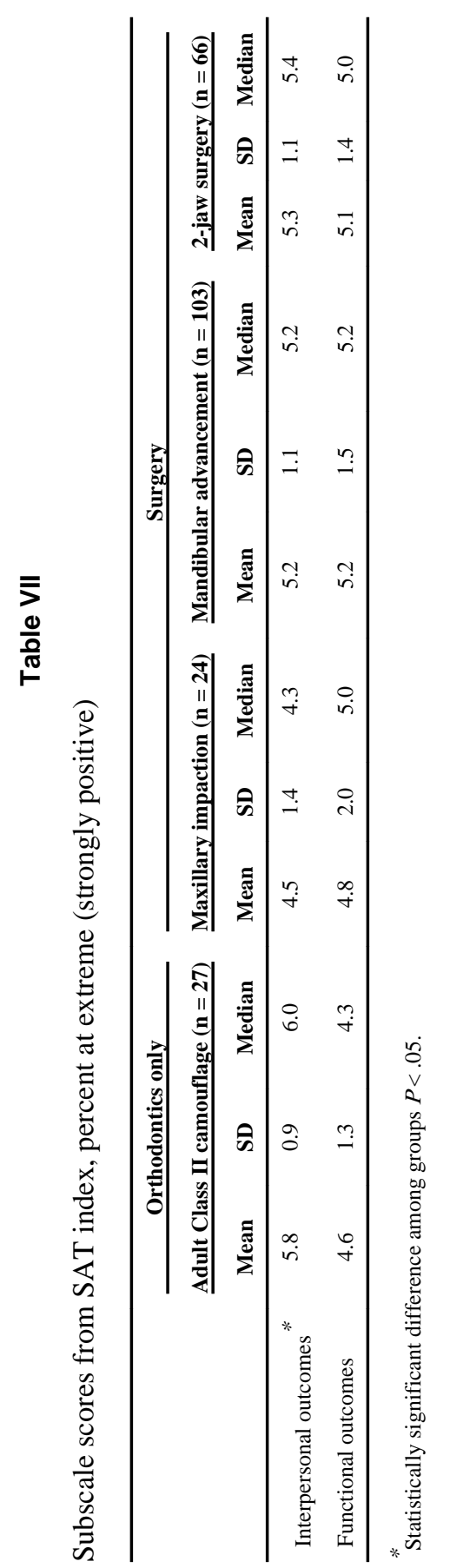




\section{Table VIII}

Questionnaire items from PSPOF index, percent at extreme (strongly positive)

\begin{tabular}{|c|c|c|c|c|}
\hline & \multirow{2}{*}{$\begin{array}{r}\text { Orthodontics only } \\
\begin{array}{r}\text { Adult Class II camouflage } \\
(\mathrm{n}=28)\end{array}\end{array}$} & \multicolumn{3}{|c|}{ Surgery } \\
\hline & & $\begin{array}{r}\text { Maxillary } \\
\text { impaction }(n=24)\end{array}$ & $\begin{array}{r}\text { Mandibular } \\
\text { advancement }(\mathrm{n}= \\
106)\end{array}$ & $\begin{array}{r}\text { 2-jaw surgery (n } \\
=70)\end{array}$ \\
\hline \multicolumn{5}{|l|}{ Occlusion and function } \\
\hline Front teeth stick out too much & 7 & 21 & 8 & 6 \\
\hline Have deep bite & 15 & 8 & 10 & 6 \\
\hline Have open bite & 11 & 25 & 21 & 21 \\
\hline Front teeth do not fit properly & 15 & 33 & 13 & 13 \\
\hline Cannot chew properly & 15 & 13 & 11 & 10 \\
\hline Posterior open bite & 11 & 9 & 12 & 14 \\
\hline Back teeth do not fit properly & 7 & 9 & 17 & 13 \\
\hline Cannot chew food well & 0 & 25 & 7 & 22 \\
\hline \multicolumn{5}{|l|}{ TMJ related problems } \\
\hline Jaw hurts when mouth open wide & 4 & 21 & 28 & 27 \\
\hline Jaw moves sideways when opening & 4 & 13 & 8 & 9 \\
\hline Jaw makes grating/grinding noise & 4 & 17 & 17 & 9 \\
\hline Cannot open mouth wide & 11 & 21 & 19 & 19 \\
\hline Jaw pops/clicks & 14 & 25 & 28 & 19 \\
\hline \multicolumn{5}{|l|}{ Pain, discomfort, soreness } \\
\hline Pain in temples & 4 & 13 & 8 & 4 \\
\hline Pain in jaw joints & 18 & 46 & 27 & 34 \\
\hline Pain in jaw muscles & 11 & 38 & 23 & 25 \\
\hline Pain in ears & 7 & 21 & 11 & 10 \\
\hline
\end{tabular}




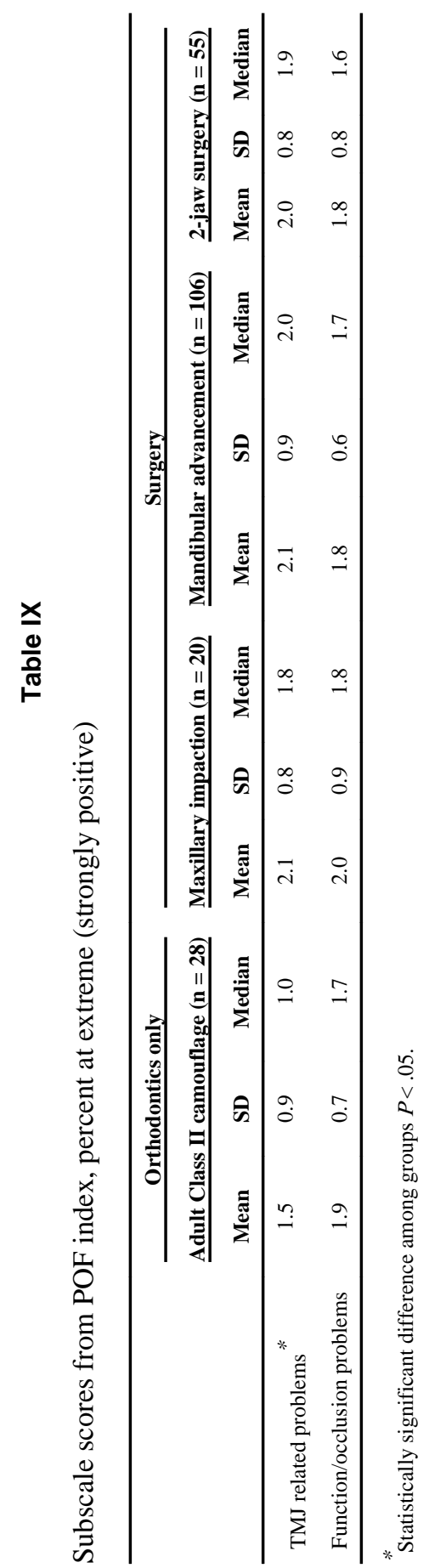


Table X

Questionnaire items from FI index, percent at extreme (strong positive feelings)

\begin{tabular}{|c|c|c|c|c|}
\hline & Orthodontics only & \multicolumn{3}{|c|}{ Surgery } \\
\hline & $\begin{array}{l}\text { Adult Class II camouflage }(\mathrm{n}= \\
\mathbf{2 8} \text { ) }\end{array}$ & $\begin{array}{l}\text { Maxillary impaction } \\
(\mathbf{n}=\mathbf{2 0})\end{array}$ & $\begin{array}{l}\text { Mandibular advancement } \\
\qquad(\mathrm{n}=\mathbf{8 2})\end{array}$ & 2-jaw surgery $(n=$ \\
\hline \multicolumn{5}{|l|}{ Cosmetic image } \\
\hline Hair & 70 & 85 & 83 & 78 \\
\hline Ears & 59 & 65 & 78 & 75 \\
\hline Forehead & 57 & 60 & 78 & 65 \\
\hline Eyes & 74 & 85 & 87 & 78 \\
\hline Nose & 52 & 58 & 66 & 64 \\
\hline Lips & 70 & 65 & 76 & 60 \\
\hline Facial Complexion & 59 & 70 & 66 & 64 \\
\hline Neck & 48 & 55 & 71 & 69 \\
\hline Eyebrows & 52 & 75 & 79 & 65 \\
\hline Cheeks/Cheekbones & 70 & 65 & 86 & 65 \\
\hline Facial skin tone & 56 & 80 & 71 & 65 \\
\hline \multicolumn{5}{|l|}{ Dentofacial image } \\
\hline Mouth & 76 & 60 & 79 & 62 \\
\hline Teeth & 74 & 65 & 82 & 67 \\
\hline Chin & 48 & 45 & 76 & 57 \\
\hline Profile & 52 & 55 & 73 & 64 \\
\hline Smile & 74 & 52 & 69 & 63 \\
\hline
\end{tabular}

*Statistically significant difference among groups $P<.05$. 


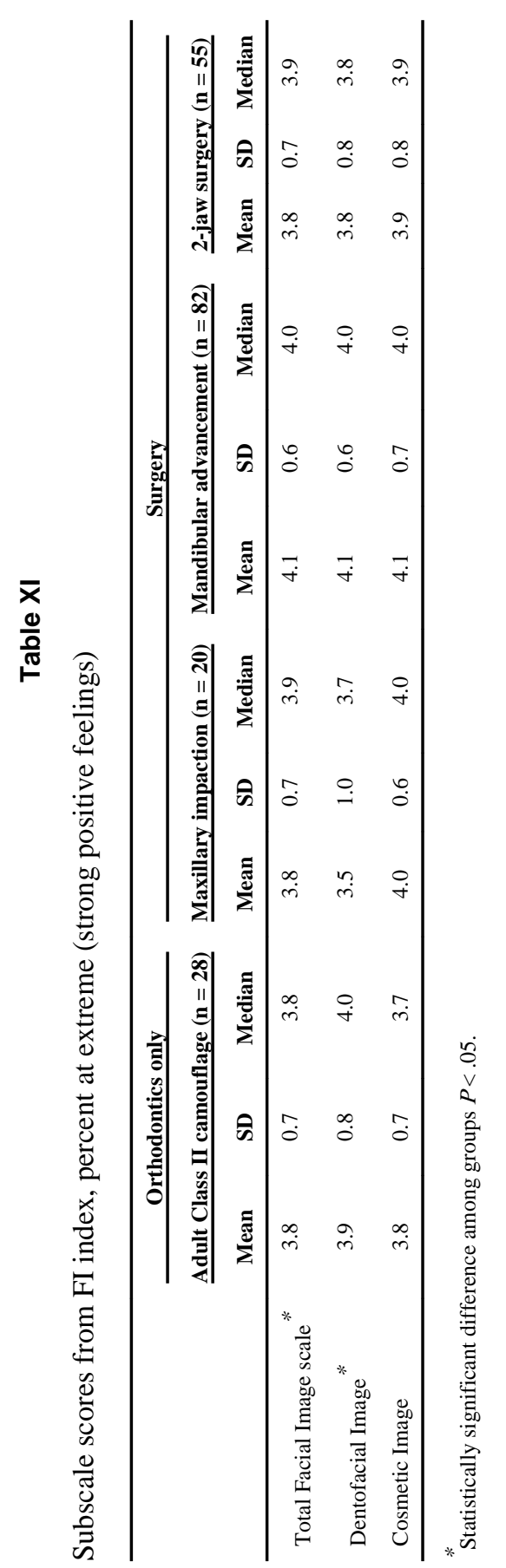

